\title{
A Novel Tree Shrew Model of Chronic Experimental Autoimmune Uveitis and its Disruptive Application Efficiency
}

\section{Kaijiao Hu}

Chongqing Medical University

Longbao LV

Kunming Institute of Zoology

Hui Huang

Chongqing Medical University

Guangnian Yin

Chongqing Medical University

Jie Gao

Chongqing Medical University

Jianping Liu

Chongqing Medical University

Yaying Yang

Chongqing Medical University

Wenxin Zeng

Chongqing Medical University

\section{Yan Chen}

Chongqing Medical University

Ni Zhang

Chongqing Medical University

Feiyan Zhang

Kunming Institute of Zoology

\section{Yuhua Ma}

Kunming Institute of Zoology

Feilan Chen ( $\nabla$ chenfl@cqmu.edu.cn )

Chongqing Medical University

\section{Research Article}

Keywords: Experimental autoimmune uveitis (EAU), Chronic, Tree shrew, Differentially expressed genes (DEG), metabolic pathways, microglia, Th1, Th17, Regulators of G-protein signaling 4 (RGS4), Dihydroartemisinin (DHA) 
Posted Date: January 18th, 2022

DOI: https://doi.org/10.21203/rs.3.rs-1259138/v1

License: (c) (i) This work is licensed under a Creative Commons Attribution 4.0 International License. Read Full License 


\section{Abstract}

Background: Previous studies have established several animal models for experimental autoimmune uveitis (EAU) in rodents without the human fovea. This study aimed to develop and disrupt the application of a novel EAU model in tree shrews with a cone-dominated retina resembling the human fovea.

Methods: Tree shrews were immunized with six inter-photoreceptor retinoid-binding proteins (IRBPs) in Freund's adjuvant. Immunized tree shrews were clinically and pathologically evaluated for the development and characteristics of EAU. IRBP-specific T-cell proliferation and serum cytokine production of tree shrews were evaluated to determine immune responses. Gene-expression profiles were analyzed in the eyes of tree shrews under EAU immunization with screened IRBP to identify differentially expressed genes (DEGs). The disruptive effects of the DEG RGS4 inhibitor CCG 203769 and dihydroartemisinin on the EAU were investigated to evaluate the potential application of tree shrew EAU.

Results: Tree shrew IRBP $1197-1211$ and bovine R14 successfully induced chronic EAU with predominate features of mild vasculitis, diffuse inflammatory infiltration, and retinal damage in the tree shrews. Immunohistological results showed that ionized calcium-binding adaptor molecule-1 (Iba-1) ${ }^{+}$ macrophages, purinergic receptor P2Y, G-protein-coupled 12 (P2RY12) ${ }^{+}$microglia, and CD11 $\mathrm{c}^{+}$dendritic cells, $\mathrm{CD} 4^{+}$and $\mathrm{CD} 8^{+}$T-cells were the main infiltrating cells in the ciliary body, retina, and choroid during the development of disease. IRBP-specific lymphocyte proliferation and pathogenic T helper (Th) 1 and Th17 responses were observed in tree shrew EAU. DEGs in the R14 group were involved in regulation of cell-substrate adhesion, adenylate cyclase activity, and ruffle, and DEGs in the IRBP 1197-1211 group were enriched in the supramolecular complex and the microtubule. In particular, the pathways of bacterial invasion of epithelial cells and inflammatory mediator regulation of TRP channels were significantly enriched in the R14 group. RGS4 inhibition and dihydroartemisinin can significantly alleviate the inflammatory severity of EAU by reducing the retinal pathological injuries after treatment.

Conclusions: Our study first established a novel chronic EAU in tree shrews elicited by R14 and IRBP 1197 1211 , probably by altering important pathways and genes related to microglia infiltration, photoreceptor damages, and T-cell responses, thereby allowing the pathogenesis and therapeutics study of the foveainvolved visual disturbance in human uveitis.

\section{Background}

Worldwide, uveitis is a group of common blinding intraocular inflammatory disorders, some of which arise with an unknown infectious trigger and are frequently associated with immune reactions to unique retinal proteins[1, 2]. Retinal folds, photoreceptor cell damages, and inflammatory infiltration into the eyeball are primary features of autoimmune uveitis and lead to vision loss in humans. Although rods outnumber cones, human vision mostly relies on cone photoreceptors since cones play an important role in sharp chromatic vision and spatial acuity [3]. In particular, the human fovea, a specialized region of the 
central retina, shows the highest density of cones and is essential for human vision [4]. Despite numerous research efforts, the etiology of uveitis remains unclear, and effective therapies need to be further identified. Experimental autoimmune uveitis (EAU) in nocturnal rodents with poor vision and non-human primates with fovea-like characteristics are utilized in research to investigate human diseases, but no animal model can fully represent the complexity of human disorders, so it is necessary to develop a variety of animal models to represent the distinct aspects and diverse findings of uveitis in humans. Tree shrews are an intermediary species between primates and rodents with excellent vision and have eye anatomical structures and immune systems similar to those of both primates and humans $[5,6]$. They have been widely used as a model animal for studying glaucoma, myopia, refractive development, and central visual processing and are becoming a crucial model animal in vision research [7-9]. Here, we developed a novel tree shrew model of uveitis by a classic induced method with screened bovine interphotoreceptor retinoid-binding protein (IRBP) R14 (BR14, aa 1169-1191) and tree shrew IRBP $1197-1211$ in complete Freund's adjuvant (CFA, Sigma-Aldrich, St. Louis, MO, USA) to trigger the immune responses characterized by ciliary injection, generalized fundus changes in reflectivity, and retinal folding and damages. Predominately, infiltrating macrophages, microglia, and dendritic cells in the retina and choroid throughout the entire course of the disease and Th1 and Th17 responses in the later stages of EAU are the main immunological characteristics. Meanwhile, the DEG characteristics in IRBP-induced and R14induced EAU are most enriched in the CAMP and cGMP biosynthetic processes, which are involved in the microglia-activation process and organelle transportation along microtubules. The inhibitor of DEG gene regulators of G-protein signaling 4 (RGS4) and Chinese herb derivative dihydroartemisinin (DHA) had a protective effect against IRBP-induced EAU by reducing the retinal pathological injuries after treatment.

\section{Results}

\section{R14 and IRBP $1197-1211$ induce ocular inflammation with chronic clinical findings in tree shrews}

To establish EAU in the tree shrew model, adult female and male mice were subcutaneously immunized with different dosages of R14, R16, and IRBP 1197-1211 peptides from bovine and tree shrew sources (Table.1). The ocular anterior and posterior segments were observed two or three times per week at day 7 post immunization. Tree shrews immunized with bovine R14 and IRBP ${ }_{1197-1211}$ from tree shrews developed chronic EAU, with onset occurring between days 5-15 and a peak happening between days 925 , followed by varying periods of remission post-immunization (Fig. 1). No obvious clinical findings were observed in tree shrews immunized with another retinal antigen. Conjunctival hyperemia and whitish hypopyon spots (Fig. 1, triangle), ciliary injection (Fig. 1, arrow), corneal edema, and corneal ulceration (Figs. $1 \mathrm{i}$ and $\mathrm{j}$, stars) were observed by slit-lamp microscopy at days 5-15 post-immunization with 50 , 300 , and $800 \mu \mathrm{g}$ of bovine R14 or IRBP $1197-1211$, lasting for a few days before disappearing, which was then followed by varying periods of remission and relapse (Fig. $1 \mathrm{~A}-\mathrm{R}$ and $\mathrm{a}-\mathrm{r}$ ). The clinical scores of

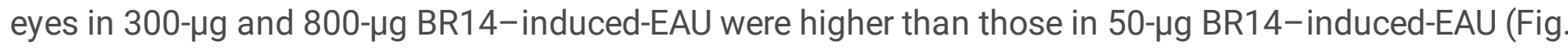


1S). There was no significant difference between the different dosages of IRBP-induced EAU (Fig.

$1 \mathrm{~s})$. The course of EAU tree shrews immunized with bovine R14 or IRBP 1197-1211 $_{1}$ was chronic and/or relapsing (Fig. $1 \mathrm{~S}-\mathrm{V}$ and $\mathrm{s}-\mathrm{v}$ ). The recurrences varied between individual eyes and animals, and the incidences of recurrence were $100 \%$ (6/6), 33\% (2/6), 100\% (4/4), 11\% (1/9), 50\% (4/8), and 50\% (4/8), respectively, in the 50- $\mu \mathrm{g}-\mathrm{BR} 14-$ induced, $300-\mu \mathrm{g}-\mathrm{BR} 14-$-induced, $800-\mu \mathrm{g}-\mathrm{BR} 14-$ induced, $50-\mu \mathrm{g}-\mathrm{IRBP}_{1197-}$ ${ }_{1211}$-induced, 300- $\mu \mathrm{g}-\mathrm{IRBP}_{1197-1211}$-induced, and 800- $\mu \mathrm{g}-\mathrm{IRBP}_{1197-1211}$-induced tree shrews after an extended observation period of 3-13 weeks post-immunization. The ratio of occurring death was 6 in 30

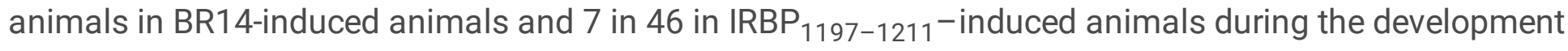
of disease. Mild vascular cuffing (Fig. 2, white arrow), retinal degeneration (Fig. 2, black arrow), a generalized change in reflectivity or hyper-reflectivity, pale optic nerve, opacification, and vascular attenuation were observed during fundus examination during at late stages of tree shrew EAU (Fig. $2 p$ and $u-x)$.

\section{Chronic and progressive pathological damages of eyes in R14-induced and IRBP-induced EAU tree shrews}

The main histopathological changes involved inflammatory infiltration (Fig. 3, triangle) into the conjunctiva, cornea, anterior chamber, ciliary processes (Fig. $3 \mathrm{I}$, triangle), choroid, and retina (Fig. $3 \mathrm{E}$ and i) as well as retina lesions (Fig. 3, stars and arrow) in the $50-\mu \mathrm{g}, 300-\mu \mathrm{g}, 400-\mu \mathrm{g}$, and $800-\mu \mathrm{g} \mathrm{Ag-induced}$ tree shrews. The retinas of EAU tree shrews were characterized by retinal folds (Fig. 3, white arrow) and detachments, retinal pigment epithelium folds, loss of the photoreceptor outer segments, formation of subretinal amorphous eosinophilic deposits (Fig. 3, stars), and abnormally long photoreceptor cell nuclei at the early and peak phases of the disease. Mild and moderate inflammatory infiltrations were observed in various retinal layers, however, the retina was more so characterized by atrophy in the retinal layer with thin photoreceptor cells, bipolar cell layers, and ganglion cell layers in the late stage (Fig. 3 o, blue arrow) and abnormal proliferative structures in the subretinal space (Fig. 3 o, circle). The histopathological damages in all dead mice were observed as early as day 7 post immunization, with an average grade of $2-3$. Histopathological scores of the inflammatory peak in 50- $\mu$-induced EAU with an average grade of 1

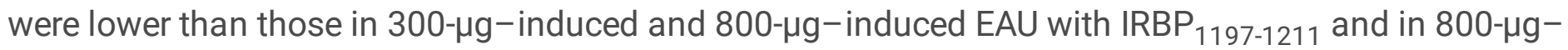
induced EAU with BR14 (Fig. 3q).

\section{Infiltration of innate immune cells and adaptive immune cells into the eyes of tree shrews with EAU}

At the peak of disease (days 25-45) induced by $50 \mu \mathrm{g}$ of BR14, $300 \mu \mathrm{g}$ of R14, $50 \mu \mathrm{g}$ of IRBP $1197-1211$, and $300 \mu \mathrm{g}$ of IRBP ${ }_{1197-1211}, \mathrm{CD} 45$ cells were observed in the choroid and the retina, notably, these cells included $\mathrm{CD} 11 \mathrm{c}^{+}$dendritic cells (DCs), $\mathrm{CD} 68^{+} / \mathrm{lba}-1^{+}$macrophages, $\mathrm{P} 2 \mathrm{RY} 12^{+}$microglia, $\mathrm{CD} 4^{+} \mathrm{T}$-cells, and CD8 ${ }^{+}$T-cells (Fig. 4). CD11 $\mathrm{C}^{+}$DCs had predominately infiltrated in the non-pigmented epithelium of the ciliary processes but scarcely infiltrated into the photoreceptor cell, ganglion cell, and corneal epithelium layers on days 26,45 , and 46 post immunization with $50 \mu \mathrm{g}$ of BR14, $300 \mu \mathrm{g}$ of R14, $50 \mu \mathrm{g}$ of IRBP $1197-$ 1211 , and $300 \mu \mathrm{g}$ of IRBP $1197-1211$. At the peak or late stages of the disease, CD $4^{+}$T-cells were distributed in the cornea, ciliary body, photoreceptor layer, inner nuclear layer, and ganglion cell layer, whereas these 
cells were absent from these areas at the onset of disease (Fig. $4 \mathrm{a}-\mathrm{i}$ ). During the peak and latter stages of the disease, $\mathrm{CD}^{+}{ }^{+}$T-cells were also scattered in the photoreceptor layer and ganglion cell layer (Fig. 4 $\mathrm{m}-\mathrm{x}$ ). Moreover, periodic acid-Schiff (PAS) staining and oil red 0 staining yielded negative results for subretinal amorphous eosinophilic deposits, but the results were partly positive following staining by lba1 (Figs. 4 F and f), P2RY12 (Fig. 5 E, J, and R), CD 8 (Fig. 4 r, t, and w), and amyloid $\beta$-protein (A $\beta$ ) (Fig. 5 j and $i)$ at the site of damage of the retina at the peak or late stages of disease.

\section{Enrichment of DEGs in tree shrews with EAU occurred mostly in adenylate cyclase activity, ruffle, and cell development}

To understand what differences existed between the eyes of tree shrews with and without EAU, DEG analysis was performed using RNA sequencing (RNA-seq). After the qualified control evaluation, the transcript expression was obtained based on fragments per kilobase million (FPKM). The clusters of DEGs in eye samples from tree shrews immunized with $800 \mu \mathrm{g}$ of BR14 or $800 \mu \mathrm{g}$ of IRBP $1197-1211$ and control healthy tree shrews showed that there was a high correlative index between the samples within the same group, however, there was a low correlative index between the samples of different groups (Figs. $5 \mathrm{~A}$ and $\mathrm{B}$ ). The heatmap revealed that the expression patterns were significantly different in the eyes of tree shrews with EAU compared to those of the control group (Figs. 6 A and B). A total of 991 DEG transcripts were identified (Fig. 6C), and the upregulated and downregulated transcripts totaled 401 and 590 , respectively, in the eyes of the BR14-induced tree shrews compared to those of the control group. The DEGs were shown to be chiefly associated with the following Gene Ontology (GO) terms: "activity," "part," "synapse," "complex," "signaling," "process," and "stimulus" The enriched GO categories in the BR14-induced group were spread across 50 biological processes, 9 cellular components, and 11 molecular functions, the top 30 enriched GO categories are mostly involved in the regulation of cellsubstrate adhesion, bone morphogenesis, regulation of body fluid levels, and the transmembrane receptor protein tyrosine kinase signaling pathway (Fig. 7A), which enabled us to further explore their potential role in EAU. Of interest, the DEGs in the regulation of body fluid levels in BR14-induced tree shrews with EAU were mostly related to the adenylate cyclase activity, which involved significantly increased LOC102483497 and significantly decreased ADCY9 and ADCY4. Meanwhile, a total of 1,046 DEG transcripts were identified (Fig. 6 D), and the upregulated and downregulated transcripts totaled 520 and 526 , respectively, in the eyes of the IRBP-induced tree shrews compared to those of the control group. The DEGs were shown to be chiefly associated with the following Gene Ontology (GO) terms: "behavior," "adhesion," "regulation," "killing," "proliferation," "organization or biogenesis," and "process". Here, the enriched GO categories were spread across 42 biological processes, 25 cellular components, and 11

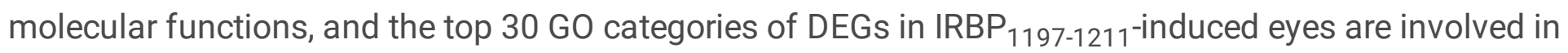
the supramolecular complex, supramolecular polymer binding, supramolecular fiber, and microtubule (Fig. 7B).

As shown in the Kyoto Encyclopedia of Genes and Genomes (KEGG) enrichment pathway of the messenger RNAs (mRNAs) (Fig. 7C and Table 2), five human diseases, including bacterial invasion of epithelial cells, endocrine resistance, pathways in cancer, amoebiasis, and shigellosis, eight 
environmental information processing, four cellular processes including inflammatory mediator regulation of TRP channels, 11 organismal systems were significantly enriched in the eye group of BR14induced tree shrews. Especially, the downregulated gene recombinant nitric oxide synthase 3 (NOS3) is involved in the PI3K-Akt signaling pathway and platelet activation. Similarly, the downregulated genes frizzled-related protein 4 (SFRP4) and sex-determining region Y-box(SOX17) are involved in the immunerelated Wnt signaling pathway (Table 3). As shown in the KEGG enrichment pathway of the mRNAs (Fig. 7D, Table 4), the AMPK signaling pathway and MAPK signaling pathway classified in environmental information processing and autophagy but classified in cellular processes, spliceosome classified in genetic information processing, methane metabolism, phosphonate and phosphinate metabolism, glycerophospholipid metabolism, biosynthesis of secondary metabolites, carbon metabolism classified in metabolism, and protein digestion and absorption classified in organismal systems-were slightly enriched in the eye group of IRBP-induced tree shrews. Of note, the significantly downregulated transcript of the Fms-related receptor tyrosine kinase 4 (FLT4) and the upregulated transcript of LOC102488698 are involved in the PI3K-Akt signaling pathway and in autophagy, respectively.

\section{Verification of DEGs by quantitative polymerase chain reaction (qPCR)}

The expression levels of six DEGs were detected by qPCR to determine the content of the DEG library. Fig. 8 shows the same expression patterns in the qPCR and RNA-seq analyses, thereby validating our results of RNA-seq.

\section{Tree shrews with EAU show antigen-specific T-cell proliferation after EAU induction with $\mathrm{IRBP}_{1197-1211}$ or BR14}

The antigen-presenting cells (APCs) and T-cells of spleen and lymph lodes from tree shrews with EAU and healthy controls were collected on day 25 or days $40-45$ post-immunization with $800 \mu \mathrm{g}$ of antigens and were in vitro co-cultured with or without antigen. The T-cell proliferation assay was determined by MTT stimulated by APCs with or without antigen for $72 \mathrm{~h}$. We observed a significantly higher proliferation of Tcells in the tree shrew EAU group immunized with $800 \mu \mathrm{g}$ of IRBP in the presence of $10 \mu \mathrm{g}$ of IRBP and immunized with $800 \mu \mathrm{g}$ of BR14 in the presence of $20 \mu \mathrm{g}$ of BR14 compared to with no antigen stimulation, respectively (Fig. $9, P<0.05$, one-way analysis of variance).

\section{Cytokine profile of peripheral blood in tree shrews with EAU}

To evaluate cytokine production of the peripheral blood of tree shrews with EAU, serum was obtained from the peripheral blood of the control healthy tree shrews and tree shrews with EAU induced with $50 \mu \mathrm{g}$ of BR14, $300 \mu \mathrm{g}$ of BR14, $800 \mu \mathrm{g}$ of BR14, $50 \mu \mathrm{g}$ of IRBP, $300 \mu \mathrm{g}$ of IRBP, or $800 \mu \mathrm{g}$ of IRBP by centrifugation. The Th17 signature cytokine interleukin (IL)-17A, Th1 signature cytokines IL-2, interferon (IFN)- $\gamma$, and tumor necrosis factor alpha (TNF-a), Th2 signature cytokines IL-4, IL-6, and IL-10 of serum in the tree shrews immunized with R14 and IRBP ${ }_{1197-1211}$ on day 25 and day 40 post immunization were detected using cytometric bead array. Regarding the protective Th2 cytokine level, the FACS results 
showed increased IL-10 levels in the 50- $\mu$ IRBP-induced group on day 25 compared to the healthy control group. For pro-inflammatory cytokine production, the 50- $\mu$ g IRBP-induced group on day 25 showed an increased Th1 cytokine TNF-a, while on day 40 the $50-\mu \mathrm{g}$ IRBP-induced group and the $800-\mu \mathrm{g}$ IRBP-induced group showed an increased IL-2 level compared to the healthy control group on days 25

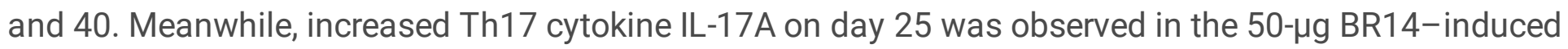
group compared to the control group (Fig. 9).

\section{Treatment of EAU induction by IRBP $_{1197-1211}$ with an RGS4 inhibitor and dihydroartemisinin (DHA) significantly ameliorates the EAU}

We then evaluated whether a DEG RGS4 inhibitor and DHA would inhibit the development of EAU. As the average EAU scores induced in $50-\mu \mathrm{g}-\mathrm{Ag}$-induced and $300-\mu \mathrm{g}-\mathrm{Ag}$-induced tree shrews were lower than

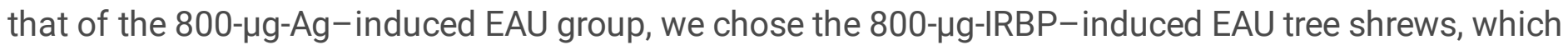
developed stronger pathological lesions using the same experimental protocol for EAU induction. Tree shrews were immunized with $800 \mu \mathrm{g}$ of $\mathrm{IRBP}_{1197-1211}$ and then intraperitoneally injected with one of corn oil, the RGS4 inhibitor CCG $203769(0.137 \mathrm{mg} / \mathrm{kg})$ and the DHA on days 0, 1, and 2 post immunizations. As shown in Fig. 10, the immunized tree shrews treated with both CCG203769 and DHA achieved a marked reduction in EAU histopathological scores (Fig. $10 \mathrm{Y}$ ) and developed significantly milder ocular inflammation than the corn oil-treated immunized tree shrews (Fig. $10 \mathrm{~A}-\mathrm{L}$ ). However, as shown in Fig. 10 $\mathrm{M}-\mathrm{X}$, the expression of $\mathrm{CD} 4^{+} \mathrm{T}$-cells and $\mathrm{CD} 8^{+} \mathrm{T}$-cells was not significantly different between the CCG 203769-treated tree shrews, the DHA-treated tree shrews, and the control-treated tree shrews (Fig. $10 \mathrm{Z}$ and a).

\section{Discussion}

We investigated the development, characteristics, and treatment of a novel EAU model enacted in tree shrews. The results indicated that bovine R14 and tree shrew IRBP ${ }_{1197-1211}$ could induce chronic and non-granulomatous inflammation in the retina and uvea with the typical characteristics seen in humans and mice. The novel tree shrew EAU model animals showed inflammatory infiltration of eyeball layers, retinal folding, multiple innate immune cells (retinal microglia, macrophages, and dendritic cells), and adaptive immune cells (CD4 ${ }^{+}$T-cells and $\mathrm{CD} 8^{+} \mathrm{T}$-cells), a significantly enriched neuron part in the $\mathrm{GO}$ function analysis, and bacteria invasion of epithelial cells in BR14-immunized eyes and AMPK signaling

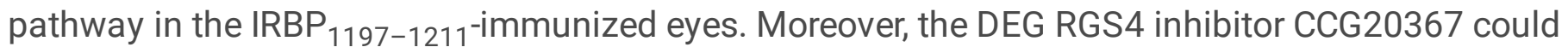
alleviate the severity of EAU by reducing the numbers of $\mathrm{CD} 4^{+}$cells on day 45 post-immunization in the 800- $\mu \mathrm{g}-\mathrm{IRBP}$-induced tree shrew eyes.

In the present study, the incidence and the intensity of the disease quantitatively relied on the respective types and doses of antigen, TB, and pertussis toxin (PTX). The mild, chronic form of the disease is established with low-dose immunization ( $<50 \mu \mathrm{g}$ of BR14 or $50 \mu \mathrm{g}$ of IRBP $_{1197-1211}, 3.5 \mathrm{mg}$ of TB and $500 \mathrm{ng}$ of PTX), while moderate- or high-dose immunization (300-800 $\mu \mathrm{g}$ of BR14 or IRBP $1197-1211$ and 
1,000 ng of PTX) induces a moderate or severe, chronic form of EAU characterized by a long duration and massive photoreceptor destruction, similar to the human condition. The lasting course of disease in the present study is similar to those of human uveitis entities such as Behçet's and Vogt-Koyanagi-Harada disease.

In humans, uveitis presents multiple distinct clinical findings, such as red and painful eyes and conjunctival injection [7-9]. In the present study, in line with the variable clinical features in human uveitis, conjunctival hyperemia, corneal ulcers, injection of the cornea, and retinal degeneration were present at various stages in the development of tree shrew EAU. Histopathological results confirmed diffuse inflammatory infiltration of the cornea, uveal, and retina, disorganization in the retinal pigment epithelium and the inner nuclear layer, and retinal folding. Those pathological features in our study can be supported by data available from studies on classical B10RIII mice [11, 12], B10.A mice, C57BL/6J mice with EAU [14], and human uveitis [15]. Those results suggest that the tree shrew EAU model in the present study can represent human uveitis and previously reported classical EAU in mice.

However, the unique features of non-granulomatous inflammation and outer segment loss or amorphous eosinophilic deposits in the inner segment of photoreceptors in the present study are distinguished from the uveitis in C57BL/6J and B10RIII mice and Lewis rats. In human and nocturnal rodents, cones constitute only a small fraction of the total number of photoreceptors, whereas, in tree shrews, cones account for $95 \%$ of the retina and rods account for $5 \%$. Ingram and colleagues demonstrated that cones have a higher ATP expenditure than rods and thereby provide the reasons why cones in the diseased retina first lose their outer segments [16-18]. In addition, the outer segment requires a tremendous amount of energy to sustain the function and structural renewal for normal homeostasis. Thus, it is not surprising that the stressors elicited by retinal antigens can further elevate metabolic demand and thereby lead to damage of the outer segment in the present study. In addition, the degeneration of their support cells, such as pigment epithelial cells, can also explain the photoreceptor dysfunction and death seen in the present study. Our observations may provide some explanation for why patients with uveitis suffer high consequences in the fovea with clustered cones when glucose transport or availability is diminished owing to the high energy requirements.

Microglia is another type of support cell-a specialized kind of macrophage-derived from yolk sacsessential for the maintenance of photoreceptor health and function and is also the primary effector of the retinal inflammatory response to acute and chronic disorders [19]. It has been demonstrated that microglia initiate the infiltration of immune cells into the retina during the early stages of EAU, and their depletion ablates the access of circulating immune cells into the retina [20]. In our present study, P2RY $12^{+}$microglia were observed at the lesions of the inner segment of photoreceptor cells and the ganglion cell layer on day 9 post-immunization with $800 \mu \mathrm{g}$ of $\mathrm{IRBP}_{1197-1211}$ in the tree shrew eye, suggesting that microglia are involved in the development of disease in tree shrews during the early phase of EAU. Previous studies have also indicated that activated microglia can interact with antigenspecific T-cells and with MHC class $\mathrm{II}^{+}$cells and CD11 $\mathrm{b}^{+}$APC cells, and they further trigger and amplify the retinal inflammation in EAU. Indeed, our immunohistochemical results further indicated that CD11 ${ }^{+}$ 
dendritic APCs, Iba- $1^{+}$macrophages, P2RY12 ${ }^{+}$microglia, CD $4^{+}$T-cells, and CD $8^{+}$T-cells present at the photoreceptor cell layer, inner nuclei layer, and ganglion cell layer in the peak and late phases of EAU. These results suggest that $\mathrm{CD} 11 \mathrm{c}^{+} \mathrm{APC}$ cells, Iba- $1^{+}$macrophages, microglia, $\mathrm{CD} 4^{+} \mathrm{T}-\mathrm{cells}$, and $\mathrm{CD} 8^{+} \mathrm{T}-$ cells are the predominant cells infiltrating into the retina and uvea. Of interest, cell debris of the inner segment at the lesions of photoreceptor cells layers positively expressed Iba-1, P2RY12, CD8, and A $\beta$ protein but was negatively stained by PAS staining and Oil $O$ staining, suggesting that macrophages/microglia and CD8 T-cells may be involved in cell debris formation by removing the photoreceptor cells damaged in response to the inflammatory stimulus. It has been demonstrated that the core marker P2RY12 of microglia is highly expressed on quiescent and activated non-inflammatory M2 microglia $[36,38]$. Under pathological conditions, microglial P2RY12 could trigger microglia motility and migration from the outer plexiform layer, inner plexiform layer, and ganglion cell layer of the retina toward the lesion site while responding to ATP release from damaged cells [21]. During this chemotaxis course, microglia adopt an amoeboid morphology, exert phagocytosis to clear the infected cells or cellular debris, and repair the damaged tissue from a ramified morphology. Moreover, P2RY12 signaling could limit retinal damage by removing potentially damaging cell debris in the context of retinal detachment, which strengthens the role of P2RY12 in inflammation [21].

The retina and choroid are primary targets of inflammatory cells attacking the development of autoimmune uveitis. In particular, the typically damaged tissues or cells in human uveitis and animal EAU are predominantly involved in the photoreceptor cell layer, blood vessels, and optic discs. It is known that photoreceptors are highly specialized neurons that contain rod and cone photoreceptors in the retina. Cones and rods morphologically are comprised of four subcellular compartments: an outer segment, inner segment, somata, and the synaptic terminal. The photoreceptor outer segment is a modified sensory cilium containing microtubular axoneme and hundreds of flattened disc-shaped membranes that provide vast light-absorbing surfaces for phototransduction [21], whereas the inner segment houses the mitochondria, which provide ATP to the outer segment and support the $\mathrm{Na}^{+} / \mathrm{K}^{+}$ATPase located in the inner segment plasma membrane [24], endoplasmic reticulum, and Golgi apparatus to meet the energy and biosynthetic needs of cells. Human and animal research has indicated that uveitis is attributed to photoreceptor damages, which predominately cause the vision loss reported in affected patients or animals. In line with previously reported studies, photoreceptor disorganization, degeneration, necrosis, and loss were observed on days 25 to 235 post-immunization with BR14 and IRBP 1197-1211 $_{\text {peptides in }}$ the present study. Of interest, the adenylate cyclase activity was significantly enriched in the eyes of BR14-induced tree shrews compared to healthy eyes. The upregulated genes, such as natriuretic peptide receptor (NPR)2, adenylate cyclase ( $A D C Y) 1, A D C Y 4, A D C Y 6, A D C Y 9$, and $L 0 C 102483497$ related to adenylate cyclase and guanylate cyclase activities in the DEGs may explain the damage mechanism of the light-sensitive photoreceptor outer segment, where phototransduction occurs, and the metabolicrelated inner segment during the development of uveitis in tree shrews. In alignment with the present results, Rao and colleagues also reported a decreased expression of ATP synthase in the early EAU retina [25]. Therefore, we propose that those upregulated genes may cause abnormal function of the 
photoreceptor layer and thus may explain the mechanism of photoreceptor damage in uveitis in tree shrews.

In particular, the gene RGS4, which can limit heterotrimeric G protein activity and promote Ga inactivation related to the regulation of the G-protein signaling domain, were significantly increased in the eyes of $I_{\text {IRBP }}$ 1197-1211_induced tree shrews compared to control eyes, suggesting that RGS4 is involved in the pathogenesis of tree shrew EAU in the current study. It has been demonstrated that RGS4 plays a significant role in various diseases, such as asthma [26], epilepsy [27], and Parkinson's disease [28], and inhibition of RGS4 with their antagonists can alleviate these diseases, suggesting that RGS proteins might be the potential therapeutic target in those conditions. Based on these facts, we further evaluated the intervention effect of the RGS antagonist CCG2037 on the development of EAU in tree shrews, and our treatment results demonstrated that inhibition of RGS4 with antagonist CCG can suppress the severity of inflammation by reducing the number of inflammatory cells such as $\mathrm{CD} 4^{+} \mathrm{T}$-cells. Although the details of the mechanism by which RGS4 contributes to uveitis await further study in the future, the findings in our study and previous reports support the notion that RGS4 may be a potential therapeutic target in uveitis and other diseases.

It has been reported that elevated levels of Th2 cytokines (IL-4 and IL-10) or mixed Th1/Th2 profiles were detected in the serum of active Behçet's patients $[29,30]$. In alignment with Behçet's patient cytokine profile, elevated protective Th2 cell IL-10 and pro-inflammatory Th1 cytokines (TNF-a and IL-2) in the serum of $50-\mu g-I_{R B P}{ }_{1197-1211}$ or $800-\mu g-I_{R B P}{ }_{1197-1211}$-induced tree shrews on days 25 and 40 were shown in our present study, suggesting a protective Th2 response and pathogenic Th1 response to counter-regulation on day 25 . In contrast, there was a shift from the mixed Th1/Th2 to pathogenetic Th2 on days 40 in tree shrew uveitis. However, in BR14-induced EAU, the findings of an increased Th17 cytokine level demonstrated that BR14-induced EAU in tree shrews is characterized by Th17 responses. Similar to in the present study, IL-17A is an important pathogenic cytokine in uveitis and EAU $[2,31]$.

\section{Conclusions}

In conclusion, the results of the current study indicated that sustained moderate uveitis can be elicited by bovine R14 and tree shrew IRBP $1197-1211$ peptides in tree shrews. This resulted in mild or moderate conjunctival hyperemia and/or ciliary injection, retinal damage, and non-granulomatous inflammatory infiltration. These changes were associated with innate immune cells (Iba- $1^{+} / \mathrm{P} 2 \mathrm{RY} 12^{+}$ macrophages/microglia, CD11 $\left.\mathrm{c}^{+} \mathrm{DCs}\right)$, adoptive immune cells (CD4 ${ }^{+}$and $\mathrm{CD} 8^{+} \mathrm{T}$-cells), and pathogenic Th1 and Th17 cytokines through multiple functions of DEGs enriched through metabolic pathways, particularly via T-cell proliferation, in tree shrews. Moreover, RGS4 inhibition could alleviate the severity of EAU by reducing the numbers of $\mathrm{CD} 4^{+}$cells in IRBP-induced EAU in tree shrews. The described tree shrew uveitis model had the potential to be a less costly platform to study the pathogenesis of and therapeutics for retina damage attributed to visual disturbance after inflammation in human uveitis. 


\section{Methods}

\section{Animals and paraffin-embedded human eye tissue}

Chinese tree shrews (Tupaia belangeri chinensis) were purchased from Kunming Institute of Zoology (KIZ), Chinese Academy of Sciences (CAS), and the Institute of Medical Biology Chinese Academy of Medical Sciences and housed in laboratory animal facilities at Chongqing Medical University. They were freely given access to chow and water. All of the procedures were carried out in compliance with the guidelines of the National Institutes of Health and the ARVO Statement for the Use of Animals in Ophthalmic and Vision Research regarding the animals' care and use. The protocol for the study (permit no. 2021031) was approved by the Ethics Committee of Chongqing Medical University. Paraffinembedded human eye tissues diagnosed as uveitis by a pathologist were obtained from the Department of Pathology, Chongqing Medical University.

\section{Immunization and treatment of EAU in tree shrews}

For induction of EAU, tree shrews 4-8 months of age were subcutaneously immunized at the base of the tail and both thighs with different immunized conditions (Table 1) according to previous studies. Briefly, peptides from $50 \mu \mathrm{g}$ to $800 \mu \mathrm{g}$ of four tree shrew R14 (aa 1169-1191) and R16 (aa 1177-1191), IRBP $_{1197-1211}$, IRBP $_{1041-1071}$, and two bovine R14 and R16 (ChinaPeptides Co., Ltd., Shanghai, China) were emulsified in a 200- $\mu \mathrm{L}$ emulsion in CFA containing or without mycobacterium tuberculosis (H37RA, ATCC 25177, American Type Culture Collection, Manassas, VA, USA). Simultaneously, PTX (Sigma-Aldrich, St. Louis, MO, USA) in $200 \mu \mathrm{L}$ was intraperitoneally injected into some animals. Four additional groups of male and female tree shrews at the age of 7-8 months were immunized with $800 \mu \mathrm{g}$ of tree shrew IRBP in a $200-\mu \mathrm{L}$ emulsion in CFA containing $500 \mathrm{mg}$ of mycobacterium tuberculosis and an additional $1 \mathrm{mg}$ of PTX to investigate the protective effect of RGS4 inhibition and dihydroartemisinin on the pathological lesions of EAU in tree shrews. 6 tree shrews were treated with $16 \mathrm{mg} / \mathrm{kg}$ dihydroartemisinin in $100-\mu \mathrm{L}$ or with the same volume of corn oil intraperitoneally every other day from days 0 to 25 or 45 post immunization. Another 6 tree shrews were treated with $0.137 \mathrm{mg} / \mathrm{kg}$ CCG 203769 in $100-\mu \mathrm{L}$ corn oil intraperitoneally every day from days 0 to 2 post immunization.

\section{Clinical and histological evaluations}

The immunized tree shrews were checked two or three times per week to observe the clinical signs from day 7 post-immunization using a slit-light scope and a funduscope. Then, immunized animals were euthanized on days $25-27,40-45$, or a humane endpoint post-immunization. Eyes of the immunized tree shrews were harvested and fixed in $10 \%$ glutaraldehyde for one hour, then immediately passed to $4 \%$ buffered formaldehyde for histopathology of the EAU. Paraffin-embedded eye tissues were sliced into 4$\mu \mathrm{m}$ sections for standard hematoxylin and eosin (H\&E) staining. Clinical and histopathological grading in mice was performed in a masked manner on a scale from 0-4 points following the previously reported criteria $[11,12]$. 


\section{RNA extraction}

A total of nine eyes from IRBP-induced EAU, BR14-induced EAU and healthy age-matched control tree shrews were collected to analyze the DEG profiles on day 26 post-immunization. The total RNA was extracted from the eyes using TRIzol@ reagent (Invitrogen, Carlsbad, CA, USA).

\section{Geneexpression profile analysis}

RNA-seq was carried out by Origingene Biomedical Technology Co., Ltd. (Shanghai, China). The mRNA was enriched from the total RNA and reverse-transcribed into complementary DNA, which was then sequenced on an Illumina Hiseq X-Ten (Illumina, Inc., San Diego, CA, USA). Raw data in the FASTQ format were analyzed with a quality evaluation using FastQC version 0.11.4 (Illumina, Inc., San Diego, CA, USA) and were filtered to obtain clean reads. The reads were mapped to the Tupaia chinensis genome (https://www.ncbi.nlm.nih.gov/genome/?term=txid246437[orgn], TupChi_1.0) using HISAT2 to obtain the total mapped reads or fragments [33]. FPKM-mapped reads values were utilized to calculate the expression of the genes in each specimen. Genes with $\|$ log2 (fold change) | 1 and a false-discovery rate $(F D R)<0.05$ were chosen as DEGs using the edger package for R $\vee 3.24$ (R Foundation for Statistical Computing, Vienna, Austria) [33]. New genes or new transcripts were identified from assembled reads with stringTie. Functional information of genes or transcripts was annotated according to the databases in NR, Swiss-Prot, Pfam, STRING, GO, and KEGG. The enrichment analysis of the GO terms and pathways was performed by traditional singular enrichment analysis. Fisher's exact test was performed for the enrichment $P$ value calculation.

\section{Quantitative polymerase chain reaction}

To validate the DEG library, the expressions of six DEGs and the endogenous control gene of actin were elucidated by qPCR in triplicate on a CFX 96 real-time PCR detection system (Bio-Rad Laboratories, Hercules, CA, USA). The primers are listed in Supplementary Table 1. The $2^{-\Delta \Delta C t}$ method was utilized to determine the relative gene expressions.

\section{T cell proliferation}

Spleens from the EAU mice in the experimental and control groups were collected on days 25-27, days $40-45$, or a humane endpoint post-immunization. Enrichment of antigen-presenting cells and T-cells in the spleens and lymph nodes were performed by passage through a nylon wool column, following the methods described in our previous study [35]. Next, $1 \times 10^{5} \mathrm{APC}$ cells and $4 \times 10^{5} \mathrm{~T}$-cells were co-cultured in a 96 -well plate with final concentrations of 0,10 , and $20 \mu \mathrm{g} / \mathrm{mL}$ of IRBP-specified antigens at $37^{\circ} \mathrm{C}$ in $5 \%$ $\mathrm{CO}_{2}$ for 72 hours. The tetrazolium salt MTT was added, and the response was detected using an automatic microplate reader (Thermo Fisher Scientific Inc., Waltham, Massachusetts, USA) at a wavelength of $450 \mathrm{~nm}$. The proliferation reaction was expressed as the mean optical density (OD) \pm standard error of the mean (SEM) of the triplicate assays. 


\section{Cytokine analysis by flow cytometry}

The peripheral venous blood was drawn into a heparinized tube from tree shrews immunized with IRBP $_{1197-1211}$ or BR14 on day 25 and day 40 post-immunization or healthy control tree shrews. Peripheral blood mononuclear cells were isolated immediately by density gradient centrifugation for T-cell subtype assay. Serum was collected from tree shrews by centrifugation for cytokine detection. Serum was utilized for flow cytometric analysis. Flow cytometry was performed with a FACS Canto II (BD Biosciences, San Jose, CA, USA). Data were analyzed with the FlowJo software (TreeStar, Ashland, OR, USA).

\section{Statistical analysis}

The experiments were performed two or three times to validate the experimental data. Statistical analysis was conducted using SPSS version 17.0 for Windows (IBM Corporation, Armonk, NY, USA). Clinical and histopathological scores were compared between the affected mice and control mice using a KruskalWallis test. An unpaired Student's $t$-test for two groups or one-way analysis of variance for three or more means was used for analysis. The level of statistical significance was set at $P<0.05$.

\section{Abbreviations}

EAU: Experimental autoimmune uveitis, IRBPs: Inter-photoreceptor retinoid-binding proteins, DEGs: Differentially expressed genes, Iba-1: Ionized calcium-binding adaptor molecule-1, P2RY12: Purinergic receptor P2Y, G-protein-coupled 12, RGS4: Regulators of G-protein signaling 4, BR14: Bovine interphotoreceptor retinoid-binding protein (IRBP) R14, CFA: Complete Freund's adjuvant, DHA: Dihydroartemisinin, DCs: Dendritic cells, PAS: Periodic acid-Schiff, Aß: Amyloid $\beta$-protein, FPKM: Fragments per kilobase million, APCs: Antigen-presenting cells, TNF-a: Tumor necrosis factor alpha, PTX: Pertussis toxin.

\section{Declarations}

\section{Acknowledgements}

We would like to thank keeper Ya Peng for the feeding of the tree shrews in this study.

\section{Authors' contributions}

F.C. and L.L. designed and performed the experiments, performed the data analysis, and wrote the manuscript. K.H., H.H., G.Y., J.G., F.Z., and Y.M. performed the experiments. K.H., H.H., and J.L. were involved in data analysis, and wrote the manuscript. J.L., Y.Y., W.Z., Y. C., and N.Z. participated in the experiments. All of the authors have given permission to be named.

\section{Funding}


The authors are grateful for the support from the National Natural Science Foundation Project (Grant no. 81670843) and the Project of Yuzhong District Science and Technology Bureau (Grant no. 20200127).

Availability of data and materials

The datasets supporting the conclusions of this article are included in the manuscript. The transcriptomic data and analysis files are openly available at BioProject database.

\section{Consent for publication}

All authors have approved the manuscript and agree with its submission.

Conflict of Interest Statement

The authors have no conflict of interest.

\section{References}

1. Caspi RR: A look at autoimmunity and inflammation in the eye. J Clin Invest 2010, 120:3073-83.

2. Zhong Z, Su G, Kijlstra A, Yang P: Activation of the interleukin-23/interleukin-17 signalling pathway in autoinflammatory and autoimmune uveitis. Prog Retin Eye Res 2021, 80:100866.

3. Fishman ES, Louie M, Miltner AM, Cheema SK, Wong J, Schlaeger NM,... La Torre A: MicroRNA Signatures of the Developing Primate Fovea. Front Cell Dev Biol 2021, 9:654385.

4. Provis JM, Dubis AM, Maddess T, Carroll J: Adaptation of the central retina for high acuity vision: cones, the fovea and the avascular zone. Prog Retin Eye Res 2013, 35:63-81.

5. Sanger F, Nicklen S, Coulson AR: DNA sequencing with chain-terminating inhibitors. Proc Natl Acad Sci U S A 1977, 74:5463-67.

6. Yao YG: Creating animal models, why not use the Chinese tree shrew (Tupaia belangeri chinensis) Zool Res 2017, 38:118-26.

7. Samuels BC, Siegwart JT, Zhan W, Hethcox L, Chimento M, Whitley R,... Girkin CA: A Novel Tree Shrew (Tupaia belangeri) Model of Glaucoma. Invest Ophthalmol Vis Sci 2018, 59:3136-43.

8. El HM, Levy AM, Gaonkar M, Gawne TJ, Girkin CA, Samuels BC,... Grytz R: Effect of Scleral Crosslinking Using Multiple Doses of Genipin on Experimental Progressive Myopia in Tree Shrews. Trans/ Vis Sci Technol 2021, 10:1.

9. Gaffney M, Cooper RF, Cava JA, Follett HM, Salmon AE, Freling S,... Carroll J: Cone photoreceptor reflectance variation in the northern tree shrew and thirteen-lined ground squirrel. Exp Biol Med (Maywood) 2021, 246:2192-201.

10. Burkholder BM, Jabs DA: Uveitis for the non-ophthalmologist. BMJ 2021, 372:m4979.

11. Xu L, Gao J, Pan Y, Tian N, He M, Jin L,... Chen F: Anti-CD40 monoclonal antibody ameliorates experimental autoimmune uveoretinitis in mice. Vet Ophthalmol 2020, 23:797-805. 
12. Caspi RR: Experimental autoimmune uveoretinitis in the rat and mouse. Curr Protoc Immuno/2003, Chapter 15:15-16.

13. Chan CC, Caspi RR, Ni M, Leake WC, Wiggert B, Chader GJ,... Nussenblatt RB: Pathology of experimental autoimmune uveoretinitis in mice. $J$ Autoimmun 1990, 3:247-55.

14. Chen Y, Chen Z, Chong WP, Wu S, Wang W, Zhou H,... Chen J: Comparative Analysis of the Interphotoreceptor Retinoid Binding ProteinInduced Models of Experimental Autoimmune Uveitis in B10.RIII versus C57BL/6 Mice. Curr Mol Med 2018, 18:602-11.

15. Burkholder BM, Jabs DA: Uveitis for the non-ophthalmologist. BMJ 2021, 372:m4979.

16. Ingram NT, Fain GL, Sampath AP: Elevated energy requirement of cone photoreceptors. Proc Natl Acad Sci U S A 2020, 117:19599-603.

17. Barone I, Novelli E, Strettoi E: Long-term preservation of cone photoreceptors and visual acuity in rd10 mutant mice exposed to continuous environmental enrichment. Mol Vis 2014, 20:1545-56.

18. Lin B, Masland RH, Strettoi E: Remodeling of cone photoreceptor cells after rod degeneration in rd mice. Exp Eye Res 2009, 88:589-99.

19. Karlen SJ, Miller EB, Burns ME: Microglia Activation and Inflammation During the Death of Mammalian Photoreceptors. Annu Rev Vis Sci 2020, 6:149-69.

20. Okunuki Y, Mukai R, Nakao T, Tabor SJ, Butovsky O, Dana R,... Connor KM: Retinal microglia initiate neuroinflammation in ocular autoimmunity. Proc Natl Acad Sci U S A 2019, 116:9989-98.

21. Muller B, Peichl L: Topography of cones and rods in the tree shrew retina. J Comp Neuro/ 1989, 282:581-94.

22. Okunuki Y, Mukai R, Pearsall EA, Klokman G, Husain D, Park DH,... Connor KM: Microglia inhibit photoreceptor cell death and regulate immune cell infiltration in response to retinal detachment. Proc Natl Acad Sci U S A 2018, 115:E6264-73.

23. Spencer WJ, Lewis TR, Pearring JN, Arshavsky VY: Photoreceptor Discs: Built Like Ectosomes. Trends Cell Biol 2020, 30:904-15.

24. Narayan DS, Chidlow G, Wood JP, Casson RJ: Glucose metabolism in mammalian photoreceptor inner and outer segments. Clin Exp Ophthalmol 2017, 45:730-41.

25. Saraswathy S, Rao NA: Mitochondrial proteomics in experimental autoimmune uveitis oxidative stress. Invest Ophthalmol Vis Sci 2009, 50:5559-66.

26. Fuentes N, McCullough M, Panettieri RJ, Druey KM: RGS proteins, GRKs, and beta-arrestins modulate G protein-mediated signaling pathways in asthma. Pharmacol Ther 2021, 223:107818.

27. Zhou H, Chisari M, Raehal KM, Kaltenbronn KM, Bohn LM, Mennerick SJ,... Blumer KJ: GIRK channel modulation by assembly with allosterically regulated RGS proteins. Proc Natl Acad Sci U S A 2012, 109:19977-82.

28. Blazer LL, Storaska AJ, Jutkiewicz EM, Turner EM, Calcagno M, Wade SM,... Neubig RR: Selectivity and anti-Parkinson's potential of thiadiazolidinone RGS4 inhibitors. Acs Chem Neurosci 2015, 6:91119. 
29. Raziuddin S, Al-Dalaan A, Bahabri S, Siraj AK, Al-Sedairy S: Divergent cytokine production profile in Behcet's disease. Altered Th1/Th2 cell cytokine pattern. J Rheumato/ 1998, 25:329-33.

30. Aridogan BC, Yildirim M, Baysal V, Inaloz HS, Baz K,... Kaya S: Serum Levels of IL-4, IL-10, IL-12, IL-13 and IFN-gamma in Behcet's disease. J Dermato/ 2003, 30:602-07.

31. Horai R, Caspi RR: Cytokines in autoimmune uveitis. J Interferon Cytokine Res 2011, 31:733-44.

32. Yin G, Zeng W, Hu K, Gao J, Liu J, Chen Y,... Chen F: Parental Uveitis Influences Offspring With an Increased Susceptibility to the Experimental Autoimmune Uveitis. Front Immunol 2020, 11:1053.

33. Kim D, Langmead B, Salzberg SL: HISAT: a fast spliced aligner with low memory requirements. Nat Methods 2015, 12:357-60.

34. Robinson MD, McCarthy DJ, Smyth GK: edgeR: a Bioconductor package for differential expression analysis of digital gene expression data. Bioinformatics 2010, 26:139-40.

35. Trizio D, Cudkowicz G: Separation of T and B lymphocytes by nylon wool columns: evaluation of efficacy by functional assays in vivo. $\mathrm{J}$ Immuno/ 1974, 113:1093-97.

\section{Tables}

Table 1. Immunization condition of different retinal antigens with various dosages 


\begin{tabular}{|c|c|c|c|c|}
\hline Type of retinal antigen & sequence & $\begin{array}{l}\text { Dosage } \\
\text { of } \\
\operatorname{Ag} \nabla \mu g \rrbracket\end{array}$ & 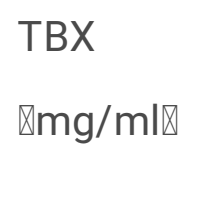 & 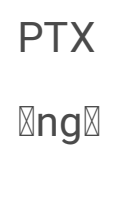 \\
\hline \multirow[t]{2}{*}{ Tree shrew R14 } & \multirow[t]{2}{*}{ PTARSVGAADGTSWEGVGVVPHV } & 100 & 1 & 0 \\
\hline & & 800 & 5 & 1000 \\
\hline \multirow{4}{*}{$\begin{array}{l}\text { Tree shrew IRBP } 1197- \\
1211\end{array}$} & \multirow[t]{4}{*}{ GAADGTSWEGVGVVP } & 50 & 5 & 1000 \\
\hline & & 300 & $3.5-5$ & 500 \\
\hline & & 400 & 5 & 1000 \\
\hline & & 800 & 5 & 1000 \\
\hline \multirow[t]{2}{*}{ Tree shrew R16 } & \multirow[t]{2}{*}{ ADGTSWEGVGVVPHV } & 50 & 5 & 0 \\
\hline & & 500 & 3.5 & 2000 \\
\hline $\begin{array}{l}\text { Tree shrew IRBP } 1041- \\
1071\end{array}$ & GYLRFDMFGDCELLTQVSELLVEHIWKKIVH & 300 & 1 & 500 \\
\hline \multirow[t]{4}{*}{ Bovine R14 } & \multirow[t]{4}{*}{ PTARSVGAADGSSWEGVGVVPDV } & 50 & 5 & 1000 \\
\hline & & 300 & $3.5-5$ & 500 \\
\hline & & 400 & 5 & 1000 \\
\hline & & 800 & 5 & 1000 \\
\hline \multirow[t]{2}{*}{ Bovine R16 } & \multirow[t]{2}{*}{ ADGSSWEGVGVVPDV } & 50 & 5 & 0 \\
\hline & & 100 & 1 & 0 \\
\hline
\end{tabular}

Table 2. Primers used for Verification of DEGs by q-PCR 


\begin{tabular}{|lll|}
\hline Gene & sequence & PCR Products (bp) \\
\hline Actin & $\begin{array}{l}\text { Forward sequence (5'-3'): CCCAGTCCTCACCCAAATACA } \\
\text { Reverse sequence (5'-3'): GGGAGACCATAAGCCTTCATAC }\end{array}$ & 183 \\
\hline RGS4 & $\begin{array}{l}\text { Forward sequence (5'-3'): GCTGCGAAACGAAGACACC } \\
\text { RIF1B }\end{array}$ & 139 \\
& Forward sequence (5'-3'): GTAGCCCCTGCCGGAATT & 198 \\
P2RY2 & Forward sequence (5'-3'): TACTACTCCTTCCGCTCACTCA & 103 \\
& Reverse sequence (5'-3'): CCAGGCAACTGTTGGCACT & \\
\hline
\end{tabular}

Table 3. The pathway enrichment of Differently Expressed Genes (DEGs) in the BR14 group 


\begin{tabular}{|c|c|c|c|}
\hline Description & Category & $P$ adjust & Gene name \\
\hline $\begin{array}{l}\text { Bacterial invasion of } \\
\text { epithelial cells }\end{array}$ & $\begin{array}{l}\text { Human } \\
\text { Diseases }\end{array}$ & 0.000265 & $\begin{array}{l}\text { XLOC_020866, MAPK15, XLOC_048568, } \\
\text { XLOC_050538, XLOC_050836, XPNPEP1, } \\
\text { PDLIM4, RBM26, SEPT9, ELMO2, DOCK1, } \\
\text { CAV1, SRC }\end{array}$ \\
\hline $\begin{array}{l}\text { Inflammatory } \\
\text { mediator regulation } \\
\text { of TRP channels }\end{array}$ & $\begin{array}{l}\text { Organismal } \\
\text { Systems }\end{array}$ & 0.000572 & $\begin{array}{l}\text { XLOC_003642, STX16, SNTA1, PPP6R2, } \\
\text { XLOC_050538, LOC102483497, P2RY2, } \\
\text { ADCY6, PRKCD, ITPR3, PRKCD, SRC, ADCY4 }\end{array}$ \\
\hline $\begin{array}{l}\text { Estrogen signaling } \\
\text { pathway }\end{array}$ & $\begin{array}{l}\text { Organismal } \\
\text { Systems }\end{array}$ & 0.000572 & $\begin{array}{l}\text { XLOC_003642, STX16, GADD45G, } \\
\text { XLOC_050538, FRMD4B, LOC102483497, } \\
\text { ATF6B, ADCY6, GABBR1, PRKCD, ITPR3, } \\
\text { ATF6B, PRKCD, SRC, ADCY4, NOS3 }\end{array}$ \\
\hline Gap junction & $\begin{array}{l}\text { Cellular } \\
\text { Processes }\end{array}$ & 0.000951 & $\begin{array}{l}\text { XLOC_003642, STX16, ZGRF1, } \\
\text { LOC102483497, TRPM1, ADCY6, } \\
\text { LOC102496850, ITPR3, SRC, ADCY4, MAP2K5 }\end{array}$ \\
\hline Platelet activation & $\begin{array}{l}\text { Organismal } \\
\text { Systems }\end{array}$ & 0.001556 & $\begin{array}{l}\text { XLOC_003642, STX16, PPP6R2, XLOC_050538, } \\
\text { XLOC_050836, LOC102483497, ARHGEF1, } \\
\text { COL1A1, CYTH3, ITPR3, SRC, ADCY4, NOS3 }\end{array}$ \\
\hline Focal adhesion & $\begin{array}{l}\text { Cellular } \\
\text { Processes }\end{array}$ & 0.002684 & $\begin{array}{l}\text { XLOC_020866, MAPK15, XLOC_048568, } \\
\text { XLOC_050538, XLOC_050836, XLOC_061360, } \\
\text { XLOC_061360, NA, ABCA4, CRYBA2, RBM26, } \\
\text { LAMB3, COL1A1, DOCK1, CAV1, SRC, FLT4, } \\
\text { PAK6 }\end{array}$ \\
\hline $\begin{array}{l}\text { Endocrine } \\
\text { resistance }\end{array}$ & $\begin{array}{l}\text { Human } \\
\text { Diseases }\end{array}$ & 0.004674 & $\begin{array}{l}\text { XLOC_003642, STX16, MAPK15, PPP6R2, } \\
\text { XLOC_050538, LOC106736342, } \\
\text { LOC102483497, ADCY6, SRC, ADCY4, MDM2 }\end{array}$ \\
\hline $\begin{array}{l}\text { GnRH signaling } \\
\text { pathway }\end{array}$ & $\begin{array}{l}\text { Organismal } \\
\text { Systems }\end{array}$ & 0.004674 & $\begin{array}{l}\text { XLOC_003642, STX16, PPP6R2, } \\
\text { LOC102483497, ADCY6, PRKCD, ITPR3, SRC, } \\
\text { ADCY4, MAP2K4 }\end{array}$ \\
\hline $\begin{array}{l}\text { Phospholipase D } \\
\text { signaling pathway }\end{array}$ & $\begin{array}{l}\text { Environmental } \\
\text { Information } \\
\text { Processing }\end{array}$ & 0.004674 & $\begin{array}{l}\text { XLOC_003642, STX16, XLOC_012724, SNTA1, } \\
\text { XLOC_050538, XPNPEP1, ZGRF1, } \\
\text { LOC102483497, RALGDS, RALGDS, CYTH3, } \\
\text { ADCY6, RAPGEF3, CYTH3, ADCY4 }\end{array}$ \\
\hline $\begin{array}{l}\text { Regulation of actin } \\
\text { cytoskeleton }\end{array}$ & $\begin{array}{l}\text { Cellular } \\
\text { Processes }\end{array}$ & 0.006887 & $\begin{array}{l}\text { ATP5ME, XLOC_020866, MAPK15, } \\
\text { XLOC_036533, XLOC_048568, XLOC_050538, } \\
\text { XLOC_050836, CDC16, XLOC_061360, ZGRF1, } \\
\text { RBM26, XLOC_086978, ARHGEF1, ACTN1, } \\
\text { DOCK1, SRC, PAK6 }\end{array}$ \\
\hline $\begin{array}{l}\text { Rap1 signaling } \\
\text { pathway }\end{array}$ & $\begin{array}{l}\text { Environmental } \\
\text { Information } \\
\text { Processing }\end{array}$ & 0.008449 & $\begin{array}{l}\text { XLOC_003642, STX16, ACO1, SNTA1, PPP6R2, } \\
\text { XLOC_050538, XLOC_050836, ZGRF1, } \\
\text { LOC102483497, XLOC_086978, RALGDS, } \\
\text { RALGDS, ADCY6, RAPGEF3, SRC, ADCY4, FLT4 }\end{array}$ \\
\hline Pathways in cancer & $\begin{array}{l}\text { Human } \\
\text { Diseases }\end{array}$ & 0.011204 & $\begin{array}{l}\text { XLOC_003642, HERC1, STX16, NXPE3, SNTA1, } \\
\text { MAPK15, XLOC_048568, XLOC_050538, } \\
\text { XLOC_050836, ZGRF1, ABCA4, } \\
\text { LOC102483497, XLOC_086978, EGLN2, } \\
\text { ARHGEF1, MECOM, SPI1, LAMB3, RALGDS, }\end{array}$ \\
\hline
\end{tabular}


RALGDS, ABL1, GNGT2, ADCY6, ADCY4, AXIN2, MDM2

Phosphatidylinositol signaling system

ErbB signaling

pathway

Amoebiasis

muscle contraction

HIF-1 signaling

pathway

Shigellosis

Adherens junction

PI3K-Akt signaling pathway ascular smooth
Environmental

Information

Processing

Environmental $\quad 0.021818$

Information

Processing

Human

Diseases

Organismal

Systems

Environmental $\quad 0.027712$

Information

Processing

Human

Diseases

Cellular

Processes

Environmental

Information

Processing

0.011361

0.025525

0.033711
XLOC_012724, SNTA1, OSBP2, XLOC_050538,

PTPRM, INPP4A, CDIPT, ITPR3

SNTA1, MAPK15, XLOC_050538,

XLOC_061360, ZGRF1, ABL1, SRC, PAK6, MAP2K4

STX16, MAPK15, XLOC_048568,

XLOC_050538, LOC102483497, RBM26, ACTN1, LAMB3, COL1A1

XLOC_003642, STX16, LOC102483497,

ARHGEF1, COL1A1, ADCY6, ITPR3, SRC, ADCY4, NOS3

AC01, SNTA1, CHMP6, XLOC_050538, ZGRF1, LSAMP, EGLN2, SERPINE1, NOS3

XLOC_020866, PPP6R2, XLOC_050836, RBM26, ELMO2, ABL1, DOCK1, SRC

XLOC_036533, BCAN, AGRN, TRPM1, RBM26, XLOC_086978, ACTN1, SRC

MAPK15, HECTD1, GADD45G, XLOC_048568, XLOC_050538, XLOC_050836, STX10, NA, CRYBA2, XLOC_086978, SGK1, ATF6B, LAMB3, GNGT2, COL1A1, ATF6B, MDM2, TEDC2, ACO1, FLT4, NOS3, ZGRF1

\begin{tabular}{lll}
$\begin{array}{lll}\text { Gastric acid } \\
\text { secretion }\end{array}$ & $\begin{array}{l}\text { Organismal } \\
\text { Systems }\end{array}$ & 0.034481 \\
\hline Axon guidance & $\begin{array}{l}\text { Organismal } \\
\text { Systems }\end{array}$ & 0.034511 \\
\hline
\end{tabular}

VEGF signaling pathway

Environmental $\quad 0.042375$ Information

Processing

Thyroid hormone synthesis

Organismal

Systems

Oxytocin signaling pathway

Organismal

Systems

0.042375

XLOC_003642, STX16, GADD45G,

LOC102483497, ATF6B, ADCY6, ITPR3, ADCY4

XLOC_003642, STX16, GALNT11,

LOC102483497, ADCY6, ITPR3, ADCY4

HEPACAM, SNTA1, MAPK15, PPP6R2, XLOC_050538, XLOC_050836, LOC102492209, XLOC_061360, NDST4, ABL1, SRC, PAK6

SNTA1, MAPK15, PPP6R2, XLOC_050538, SRC, NOS3

XLOC_003642, STX16, LOC102483497, LOC102501927, ADCY6, ITPR3, NPR2, SRC, ADCY4, NOS3, MAP2K5

Salivary secretion

Organismal

Systems

Endocrine and other Organismal

factor-regulated
Systems
XLOC_003642, STX16, GALNT11, LOC102483497, ADCY6, ITPR3, ADCY4

XLOC_003642, STX16, RUVBL2, XPNPEP1, ADCY6 
cGMP-PKG

signaling pathway
Environmental

Information

Processing
0.049559

XLOC_003642, GADD45G, XLOC_041871, BAZ1B, LOC102483497, LOC102501927, GTF2I, ADCY6, ITPR3, ATF6B, NPR2, ADCY4, NOS3

Table 4. The pathway enrichment of Differently Expressed Genes (DEGs) in the TIRBP ${ }_{1197-1211}$ group 


\begin{tabular}{|c|c|c|c|c|}
\hline Description & Category & P_value & P_adjust & Gene name \\
\hline $\begin{array}{l}\text { AMPK signaling } \\
\text { pathway }\end{array}$ & $\begin{array}{l}\text { Environmental } \\
\text { Information } \\
\text { Processing }\end{array}$ & 0.00049529 & 0.131251777 & $\begin{array}{l}\text { ADAM23, TAF15, WDR45, } \\
\text { WTIP, KMT2C, } \\
\text { XLOC_053484, } \\
\text { LOC102475736, } \\
\text { XLOC_061060, SLC10A1, } \\
\text { CREB3L2, LIPE }\end{array}$ \\
\hline $\begin{array}{l}\text { Methane } \\
\text { metabolism }\end{array}$ & Metabolism & 0.002479885 & 0.328584715 & $\begin{array}{l}\text { LOC102501767, BCL7C, } \\
\text { CHMP6, WDR45 }\end{array}$ \\
\hline Mitophagy-yeast & $\begin{array}{l}\text { Cellular } \\
\text { Processes }\end{array}$ & 0.004057935 & 0.358450945 & $\begin{array}{l}\text { LOC102488698, MAN2A2, } \\
\text { XLOC_081167, LETM1 }\end{array}$ \\
\hline $\begin{array}{l}\text { Phosphonate and } \\
\text { phosphinate } \\
\text { metabolism }\end{array}$ & Metabolism & 0.014963712 & 0.892813468 & MCRIP1, XLOC_027781 \\
\hline $\begin{array}{l}\text { Glycerophospholipid } \\
\text { metabolism }\end{array}$ & Metabolism & 0.01742543 & 0.892813468 & $\begin{array}{l}\text { LPIN1, XLOC_007834, } \\
\text { MCRIP1, PLA2G15, } \\
\text { PLA2G4F, LCLAT1, } \\
\text { XLOC_027781 }\end{array}$ \\
\hline $\begin{array}{l}\text { Protein digestion } \\
\text { and absorption }\end{array}$ & $\begin{array}{l}\text { Organismal } \\
\text { Systems }\end{array}$ & 0.024345947 & 0.892813468 & $\begin{array}{l}\text { SLC8A1, LOC102474421, } \\
\text { COL4A3, RHPN1, DNPEP, } \\
\text { COL7A1 }\end{array}$ \\
\hline $\begin{array}{l}\text { MAPK signaling } \\
\text { pathway- fly }\end{array}$ & $\begin{array}{l}\text { Environmental } \\
\text { Information } \\
\text { Processing }\end{array}$ & 0.028411711 & 0.892813468 & $\begin{array}{l}\text { FRYL, STRN4, STS, } \\
\text { XLOC_023302, TEC, } \\
\text { MAP2K4, PTPN11, SRC }\end{array}$ \\
\hline $\begin{array}{l}\text { Biosynthesis of } \\
\text { secondary } \\
\text { metabolites }\end{array}$ & Metabolism & 0.031052067 & 0.892813468 & $\begin{array}{l}\text { LPIN1, XLOC_007834, } \\
\text { NFX1, LOC102501767, } \\
\text { LOC102491763, PLA2G4F, } \\
\text { LCLAT1, CHMP6, } \\
\text { LOC102495698, } \\
\text { XLOC_027781, } \\
\text { LOC102480703, } \\
\text { SERTAD2, TAF15, WDR45, } \\
\text { FDPS, OGDH, MVD }\end{array}$ \\
\hline Spliceosome & $\begin{array}{l}\text { Genetic } \\
\text { Information } \\
\text { Processing }\end{array}$ & 0.039902368 & 0.892813468 & $\begin{array}{l}\text { XLOC_035902, CDK10, } \\
\text { DDX5, PQBP1, SLU7, } \\
\text { GSKIP, XLOC_063251, } \\
\text { XLOC_066578, LZTS3, } \\
\text { LOC102469433, }\end{array}$ \\
\hline Carbon metabolism & Metabolism & 0.044057597 & 0.892813468 & $\begin{array}{l}\text { NFX1, LOC102501767, } \\
\text { BCL7C, CHMP6, } \\
\text { SERTAD2, WDR45, OGDH }\end{array}$ \\
\hline
\end{tabular}

\section{Supplementary}

Supplementary Table 1 is not available with this version 


\section{Figures}

\section{Figure 1}

\section{Representative clinical signs and scores of tree shrew EAU induced by R14 or IRBP ${ }_{1197-1211}$.}

Major clinical signs, including conjunctival hyperemia, ciliary injections (arrow), whitish hypopyon spots (triangle), corneal ulcers (black star), and corneal edema (white star), were observed in tree shrews immunized with different dosages of BR14 (A-R) or IBRP ${ }_{1197-1211}(a-r)$. Average clinical scores per eye

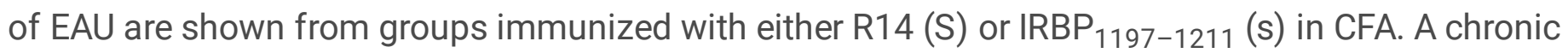
and/or relapsing course of three representative animals is shown, specifically the time courses of clinical disease from tree shrews immunized with $50 \mu \mathrm{g}$ of BR14 (T), $300 \mu \mathrm{g}$ of BR14 (U), $800 \mu \mathrm{g}$ of BR14 (V), 50 $\mu \mathrm{g}$ of IRBP $\operatorname{IRT}_{1197-1211}(\mathrm{t}), 300 \mu \mathrm{g}$ of $\operatorname{IRBP}_{1197-1211}(\mathrm{u})$, and $800 \mu \mathrm{g}$ of $\operatorname{IRBP}_{1197-1211}(\mathrm{v})$. The curves show the inflammation of eyes checked by slit-lamp biomicroscopy according to previously described criteria. Green circle, right eye, black circle, left eye, D, day post-immunization.

\section{Figure 2}

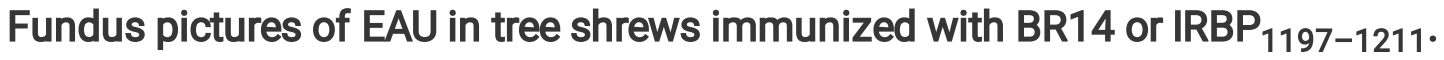

Retinal vessels with mild cuffing (black arrow), retinal degeneration (white arrow), generalized changes in reflectivity or hyper-reflectivity, pale optic nerve, opacification, and vascular attenuation were observed during fundus examination in the late stage of tree shrew EAU induced with $300 \mu \mathrm{g}$ of $\mathrm{IRBP}_{1197-1211}(\mathrm{~m}-$ p) and $800 \mu \mathrm{g}$ of $\operatorname{IRBP}_{1197-1211}(\mathrm{q}-\mathrm{x})$. D, day post immunization.

\section{Figure 3}

\section{Histological pictures of human uveitis and EAU in tree shrews immunized with BR14 or IRBP 1197-1211.}

The retinas of tree shrews with EAU were characterized by retinal folds (long arrow) and detachments, forms of subretinal amorphous eosinophilic deposits (stars), inflammatory exudation (triangle), and corneal lesions (short arrows) in the early, peak, and late phases. Atrophy (blue short arrow) and abnormal structures (white circle) in the retinal layer with thin photoreceptor cells, bipolar cell layers, and ganglion cell layers observed during the late stage. $L$ shows the eye tissue of healthy control tree shrews. $\mathrm{HU}$ shows human uveitis in the retina and ciliary body ( $\mathrm{d}$ and $\mathrm{h}$ ). $\mathrm{b}$ and $\mathrm{h}$ show the changes in the retina and ciliary body. D, day post-immunization. 


\section{Figure 4}

\section{Immune phenotypes of EAU in tree shrews immunized with BR14 or IRBP1197-1211 and human uveitis}

Iba-1 was mostly expressed on the wing cells and basal cells of the cornea, on the non-pigmented epithelium of the ciliary processes, on the choroid layer, on the visual cell layer, inner nuclear layer, and ganglion cell layer $(A-L)$. CD11c was predominately expressed on the non-pigmented epithelium of the ciliary processes and sparsely expressed on the photoreceptor cell layer, ganglion cell layer, and corneal epithelium layer $(\mathrm{M}-\mathrm{X})$. Positive expression of CD4 was observed in the cornea and various layers of the retina $(a-l)$. Weak and sparse expression of CD8 was found in the cornea and various layers of the retina $(\mathrm{m}-\mathrm{x})$.

\section{Figure 5}

\section{The expressions of P2RY12 and A of human uveitis and tree shrew EAU.}

P2RY12 was expressed on the cornea, but not on the retina, on day 18 post-immunization with $50 \mu \mathrm{g}$ of BR14 (A-B) and day 235 post-immunization with $800 \mu \mathrm{g}$ of IRBP (T-V). On day 26 post-immunization with $50 \mu \mathrm{g}$ of BR14, P2RY12 was weakly expressed on the cornea and retina (C-D). However, strong expression of P2RY12 was observed on the cornea and various layers of the retina on day 87 postimmunization with $50 \mu \mathrm{g}$ of BR14 and days $26,45,87$, and 235 post-immunization with $50 \mu \mathrm{g}$ of BR14 (C-D), $300 \mu \mathrm{g}$ of BR14 (F-J), $50 \mu \mathrm{g}$ of IRBP (K-0), $300 \mu \mathrm{g}$ of IRBP (P-S), $800 \mu \mathrm{g}$ of IRBP (T and U), and human uveitis $(\mathrm{V}-\mathrm{X})$. The positive expression of $A \beta$ was not observed in the retinal lesions on day 18 post-immunization with $50 \mu \mathrm{g}$ of BR14 (a), but weak expression was observed in the ciliary processes, retinal layer, and especially retinal lesions on day 45 post-immunization with $50 \mu \mathrm{g}$ of BR14 animals (bc), on days 26 ( $d-e$ ) and 45 (f) post-immunization with $300 \mu \mathrm{g}$ of BR14, on days 9 (g) and 235 (h-i) postimmunization with $800 \mu \mathrm{g}$ of IRBP, and in the human retina (j).

\section{Figure 6}

\section{Heatmap and volcano plot of the DEGs in different samples.}

The heatmap indicates that the expression patterns were significantly different in the eyes of BR14induced animals (A) and IRBP-induced animals (B) compared to those of the control group. BR: Eyes in the BR14-induced group. IRBP: Eyes in the IRBP-induced group. C: Eyes in the healthy control group. (C) A volcano plot of the DEGs shows 401 upregulated genes (red dots) and 590 downregulated genes (blue dots) in the eyes of the BR14-induced group compared to the control group. (D) A volcano plot of the 
DEGs shows 520 upregulated genes (red dots) and 526 downregulated genes (blue dots) in the eyes of the IRBP-induced group compared to the control group.

\section{Figure 7}

\section{The pathway enrichments of the Gene ontology (GO) and the KEGG of the DEGs.}

(A) indicates that GO of the 991 DEGs in the BR14-induced group is involved in 20 biological processes, 13 cellular components, and 9 molecular functions. (B) indicates that GO of the 1046 DEGs in the IRBPinduced group is involved in 22 biological processes, 16 cellular components, and 8 molecular functions. The lower and upper abscissae show the numbers and ratios of annotated DEGs, respectively. The ordinate shows the categories of differential expression genes according to biological process, cellular components, and molecular function. (C) indicates that the DEGs in the BR14-induced group were enriched in 5 human diseases, 4 cellular processes, and 11 organismal systems by enrichment analysis of the KEGG pathway. (D) indicates that the DEGs were enriched in 5 human diseases, 4 cellular processes, and 11 organismal systems by enrichment analysis of the KEGG pathway. The abscissa represents the name and class of the KEGG pathway enrichment of the DEGs. The ordinate represents the enrichment of the ratio calculated by the formula of enrichment ratio = sample number $/$ background number). ${ }^{\star \star \star} F D R<0.001,{ }^{\star \star} F D R<0.01$, and $* F D R<0.05$ from $\mathrm{R}$ package edge.

\section{Figure 8}

\section{Validation of the DEGs in RNA-seq by qPCR assay.}

(A) shows the expression level of DEGs by qPCR. (B) shows the expressions of DEGs by RNA-Seq. Data were expressed as the value of log2 fold-change (log2FC) relative to the control group.

\section{Figure 9}

\section{Tree shrews with EAU showed a systemic IRBP-specific immune response in vivo and in vitro.}

(A) Lymphocytes from the spleen of EAU tree shrew induced with $800 \mu \mathrm{g} \mathrm{BR} 14$ and $800 \mu \mathrm{IRBP}_{1197-1211}$ were collected on day 25 postimmunization and were stimulated with or without the BR14 and IRBP peptides. The proliferative response was measured via the Brdu assay. The affected tree shrew showed the Ag-specific proliferative response. Data are expressed as mean $\pm S E M$ and are representative of two independent experiments. ${ }^{*} P<0.05,{ }^{*} P<0.01$ in one-way ANOVA. Three tree shrews were used in each group. (B) indicates that increased IL-10 levels in the 50- $\mu$ IRBP-induced group on day 25. (C) indicates 
the 50- $\mu$ g IRBP-induced group on day 25 showed an increased Th1 cytokine TNF-a, while on day 40 the $50-\mu \mathrm{g}$ IRBP-induced group and the 800- $\mu$ IRBP-induced group showed an increased IL-2 level. (D) shows increased Th17 cytokine IL-17A on day 25 in the 50- $\mu$ g BR14-induced group. Data are expressed as mean $\pm S E M$. ${ }^{\star} P<0.05$ in one-way ANOVA. Three tree shrews were used in each group.

\section{Figure 10}

\section{RGS4 inhibition and DHA significantly ameliorated EAU.}

The immunized tree shrews treated with both CCG203769 and DHA developed significantly milder ocular inflammation (CCG203769-treated group: A-D, DHA-treated group: E-H, oil-treated group: I-L) and achieved a marked reduction in EAU histopathological score $(F)$ than the corn oil-treated immunized tree shrews on day 25 and 45 day post immunization. The expression of CD4 T-cells (CCG203769-treated group: $U$ and V, DHA-treated group: $R$ and V, oil-treated group: $M$ and $N$ ) and CD8 T-cells (CCG203769treated group: $\mathrm{K}$ and $\mathrm{L}, \mathrm{DHA}$-treated group: $\mathrm{S}$ and T, oil-treated group: $\mathrm{O}$ and $\mathrm{P}$ ) was not significantly different between the CCG 203769-treated tree shrews, the DHA-treated tree shrews, and the controltreated tree shrews $(Z, a)$. 